Ann. Biol. anim. Bioch. Biophys., I97I, 11 (4), 695-704.

\title{
TENEURS EN SÉLENIUM DANS LES TISSUS DES RUMINANTS
}

\author{
M. HIDIROGLOU, K. J. JENKINS et I. HOFFMAN* \\ Ministère de l'Agriculture du Canada \\ Institut de Recherches zootechniques, Ottawa (Canada)
}

\section{RÉSUMÉ}

Le but de ce travail a été de connaître les teneurs tissulaires en sélénium chez les ruminants après administration de cet oligo-élément.

Dans une étude pilote, on s'est servi de $\mathrm{r}_{4}$ bouvillons. Parmi eux, 6 ont reçu une seule fois, par voie intramusculaire, une préparation de sélénium (30 $\mathrm{mg}$ ) alors que six autres en recevaient 2 fois. Deux bouvillons ont servi de témoins.

Il en ressort que la viande des animaux tués 6 semaines après l'administration d'une seule dose pourrait être considérée comme salubre, alors que ceux à qui l'on avait administré 2 doses demandaient un laps d'attente de I $_{5}$ semaines.

Dans une autre expérience, 32 veaux ont été répartis en 4 lots de 8 animaux chacun. Deux lots ont reçu $4 \mathrm{mg}$ de sélénium administré oralement ou en combinaison avec de la vitamine $\mathrm{E}$. Sur les 2 lots restants, l'un était traité à la vitamine $\mathrm{E}$, et l'autre non traité.

Il en résulte que, 4 semaines après leur traitement au sélénium, les veaux pouvaient être livrés à la consommation humaine.

En outre, les teneurs en sélénium tissulaire ont été mesurées sur I 8 agneaux nés de brebis non traitées ou implantées au sélénium incorporé dans une colle aux silicones. Les résultats obtenus montrent que cette nouvelle technique d'administration de sélénium permet de prévenir la dystrophie musculaire tout en n'affectant pas la salubrité des viandes de ces agneaux.

Par ailleurs, des mesures du sélénium ont été effectuées sur les tissus de I 6 brebis implantées ou non et nourries avec un foin ayant une faible teneur en sélénium, et chez six autres brebis (normales) au régime foin et concentré à teneur normale en sélénium. On n'a observé aucune différence dans la concentration en sélénium des différents tissus entre brebis normales ou traitées. Cependant, au lieu d'implantation, il y a eu une certaine accumulation de sélénium, même 2 mois après l'administration de cet oligo-élément.

\section{INTRODUCTION}

Si l'administration de sélénium est couramment pratiquée au Canada, à titres préventif et curatif contre la dystrophie musculaire des veaux, son emploi, compte tenu des risques de toxicité, est néanmoins strictement réservé aux vétérinaires.

* Adresse actuelle : Conseil national de Recherches du Canada. 
Il en est de même dans d'autres pays. Ainsi, en Italie, Dotra, Barbo et Guarda (I964) ont constaté que l'emploi du sélénium dans le traitement de la myodystrophie enzootique du veau (DM) dans le Piémont a donné d'excellents résultats. Il en a été de même en France où LaMAND (I965) a rapporté que dans les exploitations affectées par cette maladie, une prophylaxie au sélénium pratiquée sur les veaux avait efficacement protégé ces derniers contre la DM.

Dans des études antérieures, nous avons rapporté des données sur l'absorption et la distribution du sélénium chez le Veau (HIDIRogrou, JENkINS et Carson, r969). Il va de soi que cette étude était indispensable en vue d'évaluer une substance destinée à être administrée aux animaux et, par leur intermédiaire, à la consommation humaine.

Vu sous cet aspect, nous avons pensé que ce travail devrait être complété chez les bovins, soit par une étude pilote sur le taux de persistance du sélénium en fonction du temps après son administration dans les différents tissus comestibles, soit par une comparaison des concentrations tissulaires du sélénium sur un certain nombre de veaux, traités ou non avec cet élément.

Dans une étude antérieure portant sur l'implantation de sélénium à des brebis gestantes (HIDIROGLOU et al., I97I), nous avons rapporté les effets bénéfiques de cette prophylaxie contre la dystrophie musculaire de leurs agneaux.

Il va de soi que nos résultats devaient être complétés par des analyses de sélénium tissulaire, aussi bien des agneaux que des brebis implantées, en vue de juger la salubrité de leur viande, ce qui a été l'objet d'une partie de cette étude.

\section{EXPÉRIENCE SUR BOUVILLONS IA}

La raison d'administrer du sélénium à des bouvillons qui, en principe, sont considérés comme peu atteints par la DM, est due au fait que la dégénérescence musculaire a été signalée en France chez des bouvillons de ro mois (FLAchat et al., I967; DeCAIE, I969) et récemment au Canada, dans la province de 1'Ontario (DoIG, I970).

\section{Matériel et méthodes}

Les recherches ont été effectuées sur un lot de 14 bouvillons de la race Shorthorn, tous nés et élevés dans le nord ontarien où la dystrophie musculaire sévit à l'état endémique.

Six bouvillons ont reçu par voie intramusculaire une injection de Io $\mathrm{ml}$ d'une préparation aqueuse de sélénium et de vitamine E. Cette préparation contenait $3 \mathrm{mg}$ de sélénium sous forme de sélénite de sodium et 136 UI d'acétate d' $\alpha$-tocophérol par $\mathrm{ml}$.

Une dose égale de cette même préparation a été administrée 2 fois à un mois d'intervalle à 6 autres bouvillons.

Les 2 animaux restants ont servi de témoins, un bouvillon étant abattu au début de l'expérience alors que l'autre l'était à la fin.

Le poids initial de ces animaux, nourris uniquement au foin, dont la teneur moyenne en sélénium était de $24 \mathrm{ng} / \mathrm{g}$ de matière sèche, était de $320 \mathrm{~kg}$. Le poids moyen des animaux abattus à la fin de l'expérience s'élevait à $460 \mathrm{~kg}$.

Après l'abattage de ces bouvillons, plusieurs prélèvements de tissus ont été effectués et les échantillons ont été conservés à $-20^{\circ} \mathrm{C}$.

Le sélénium a été dosé par la méthode de Hoffman, Westerby et Hidiroglou (Ig68) pour les tissus de cette expérience et de celles qui suivront. 


\section{Résultats et discussion}

Les valeurs observées dans cet essai pilote et présentées dans le tableau I ne donnent tout au plus que des ordres de grandeur. En effet, comme dans des expériences similaires de toxicologie avec de gros animaux de boucherie, vu leur coût prohibitif (SUTHERLAND, I969), un nombre restreint d'animaux a été utilisé pour étudier le taux d'élimination du sélénium dans les différents tissus d'animaux traités.

En général, les teneurs en sélénium des tissus étaient inférieures chez les animaux traités I fois par rapport à ceux traités 2 fois.

$\mathrm{Si}$, au bout de I5 semaines après l'administration d'une seule dose de sélénium, les concentrations du sélénium dans certains tissus étaient supérieures à celles du témoin abattu au début de l'expérience, elles restaient néanmoins de même ordre de grandeur dans les tissus du témoin abattu à la fin de l'expérience.

Chez les animaux ayant reçu une double dose 18 semaines après 1'administration de cet élément, les concentrations de sélénium observées restaient encore supérieures à celles des témoins. Chez ces derniers, des différences individuelles marquées en sélénium tissulaire ont été notées.

L'examen des teneurs en sélénium des différents muscles montre des concentrations plus élevées dans ceux de la cuisse, suivis de l'épaule. Cette particularité est due à l'échantillonnage de ce tissu. En effet, les masses musculaires contenant plus de gras sont moins riches en sélénium. Il est à remarquer que LINDBERG (I968) a rapporté que la portion blanche du muscle semi-tendineux chez le Porc contenait moins de sélénium que la portion rouge.

Peu de données existent dans la littérature sur les teneurs en sélénium des différents tissus chez les bouvillons normaux. MAAG et GLEN (1967) ont trouvé que la teneur moyenne en sélénium du foie de six bouvillons nourris au foin de luzerne contenant IIo ng/g s'élevait à II5 ng/g de tissu frais. Cette valeur est proche de 55I ng/g (MS) trouvée sur le foie de l'un de nos bouvillons témoins. Si l'on tient compte du fait que cet organe est le lieu de prédilection pour l'accumulation du sélénium, les teneurs en sélénium des foies des bouvillons traités une seule fois et tués à six semaines ou de ceux traités deux fois et tués à quinze semaines peuvent être considérées comme normales.

La salubrité des animaux précités peut être confirmée par les résultats obtenus par MORRIS et LEVANDER (I970) aux Etats-Unis, qui se sont servis de notre méthode analytique et ont trouvé les teneurs en sélénium ci-après exprimées en $\mathrm{ng} / \mathrm{g}$ dans les produits frais des abats et viandes du marché de Maryland États-Unis).

$$
\begin{array}{lrll}
\text { Foie } & 454 \text { à } & 409 \\
\text { Rein } & \text { I } 4 \text { Io à } & \text { I } 690 \\
\text { Roulle } & 208 \text { à } & \text { I } 89
\end{array}
$$

\section{EXPÉRIENCE SUR VEAUX $I_{B}$}

\section{Matériel et méthodes}

Ce travail a porté sur 32 veaux Shorthorn répartis dans différents lots expérimentaux qui figurent au tableau 2. Ces veaux sont nés et ont été élevés dans le nord ontarien. Les mères de ces veaux ont été nourries exclusivement, pendant leurs gestation et lactation, au foin dont les teneurs en sélénium étaient de io et $30 \mathrm{ng} / \mathrm{g}$ de matière sèche. 
M. HIDIROGLOU, K. J. JENKINS. I. HOFFMAN

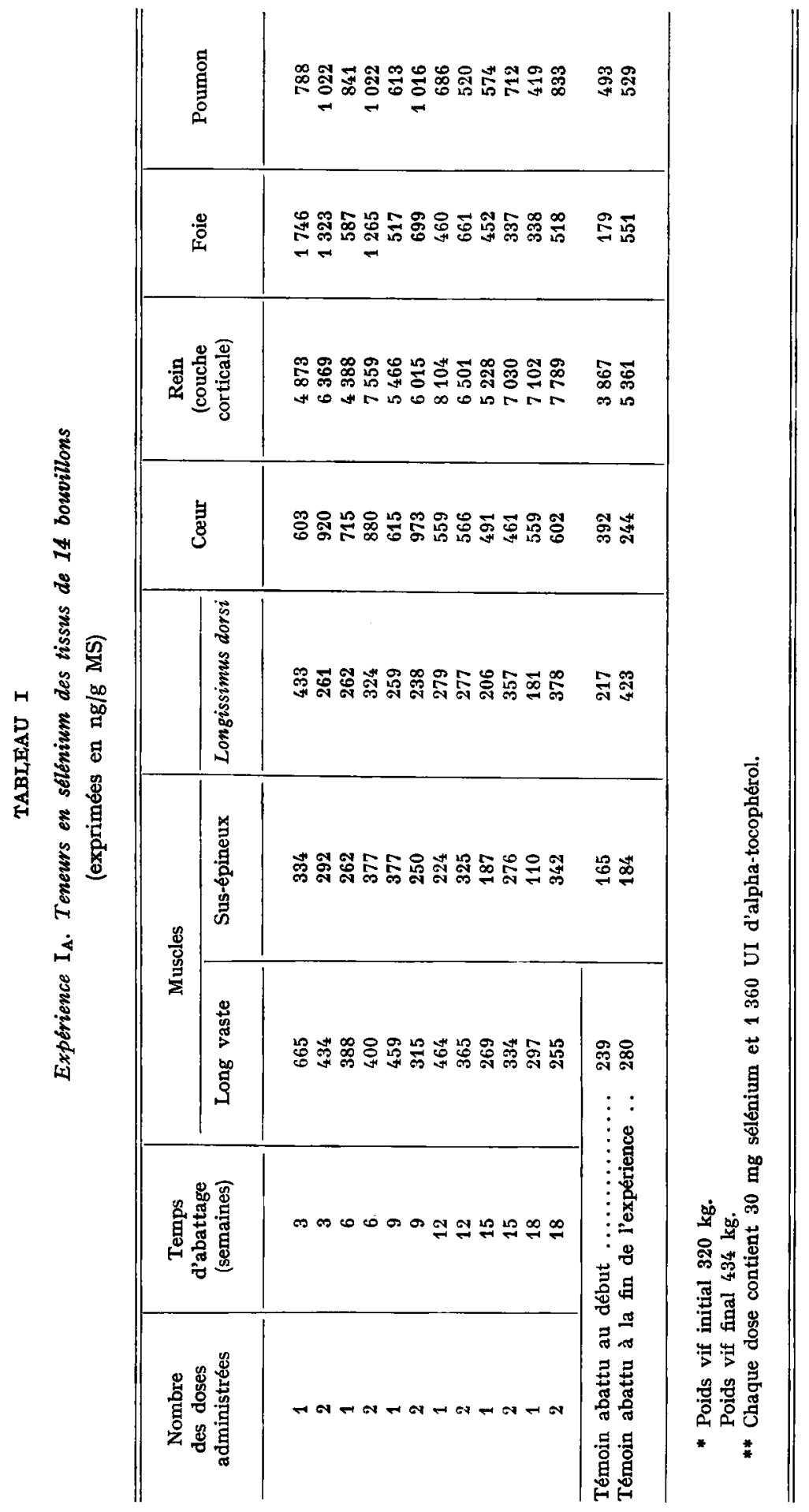


On a en outre, mis à la disposition de ces animaux un mélange minéral contenant 60 p. I0o de sel iodé et $40 \mathrm{p}$. roo de poudre d'os. Ce mélange minéral contenait 88 o0o UI de vitamine A et 13000 UI de vitamine D par kilogramme.

Ces veaux avaient au préalable reçu les traitements ci-après :

I. Pas de traitement (Veaux-témoins).

2. Administration orale à 2 et 16 jours de $4 \mathrm{mg}$ de séléniate de sodium.

3. Administration orale aux âges précités de 250 UI DL-alpha-tocopheryl-acétate.

4. Administration orale aux mêmes âges d'un mélange de $25^{\circ}$ UI de DL-alpha-tocophérylacétate additionné de $4 \mathrm{mg}$ de séléniate de sodium.

Dans chacune de ces 4 catégories, 4 veaux ont été abattus à 4 semaines et 4 autres à 8 semaines.

\section{Résultats et discussion}

D'après le tableau 2, l'administration du sélénium chez le veau a entraîné de façon notable une augmentation de son sélénium tissulaire.

Si l'on prend en considération l'âge des sujets, dans tous les traitements, les teneurs en sélénium des poumons et de la rate ont été les plus hautes et ont été plus élevées à 4 semaines qu'à 8 semaines. Nous n'navons toutefois noté une diminution sensible de la teneur en sélénium du foie que chez les sujets qui 'avaient reçu le traitement au séléniate.

Dans un mémoire précédent (HIDIROGLou et al., I967), nous avons rapporté, d'une part, les teneurs en sélénium dans les tissus des veaux sains élevés dans une région indemne de DM, et d'autre part, les teneurs en sélénium des sujets affectés de DM dans le nord ontarien.

Tout ceci permet d'appuyer l'argumentation ci-après : les teneurs en sélénium tissulaire chez les sujets traités au sélénium dans la présente étude sont du même ordre de grandeur que celles des veaux sains élevés dans une région indemne de DM, alors que les veaux non traités présentent des teneurs en sélénium comparables à celles des sujets affectés de DM.

Ces constatations nous amènent à conclure que, 4 semaines après leur traitement au sélénium, les veaux pourraient être livrés à la consommation humaine.

\section{EXPÉRIENCE SUR AGNEAUX $2_{A}$}

\section{Matériel et méthodes}

L'ensemble des méthodes a déjà été décrit (Hidiroglou, Hoffman et Jenkins, I971).

Les grandes lignes du protocole expérimental sont exposées ci-dessous.

Des brebis nourries depuis deux ans avec un foin ayant une faible teneur en sélénium (ro$25 \mathrm{ng} / \mathrm{g}$ ) ont été réparties en trois lots.

Lot I Témoins.

Lot 2 Brebis implantées au $3^{\theta}$ mois de gestation avec une préparation contenant $22 \mathrm{mg}$ de sélénium.

Lot 3 Brebis implantées avec la préparation précédente et à la même dose, mais à la fin de la gestation.

Ces implantations ont été préparées, pour le lot 2, à base d'une colle aux silicones, alors que pour le lot 3 on se servait d'huile d'arachide préalablement hydrogénée.

A chaque fois, 2 agneaux des 3 différents groupes, âgés respectivement de 60,90 et 120 jours, ont été abattus, soit au total 18 agneaux, et les divers tissus ont été analysés pour leur teneur en sélénium. 
M. HIDIROGLOU, K. J. JENKINS, I. HOFFMAN

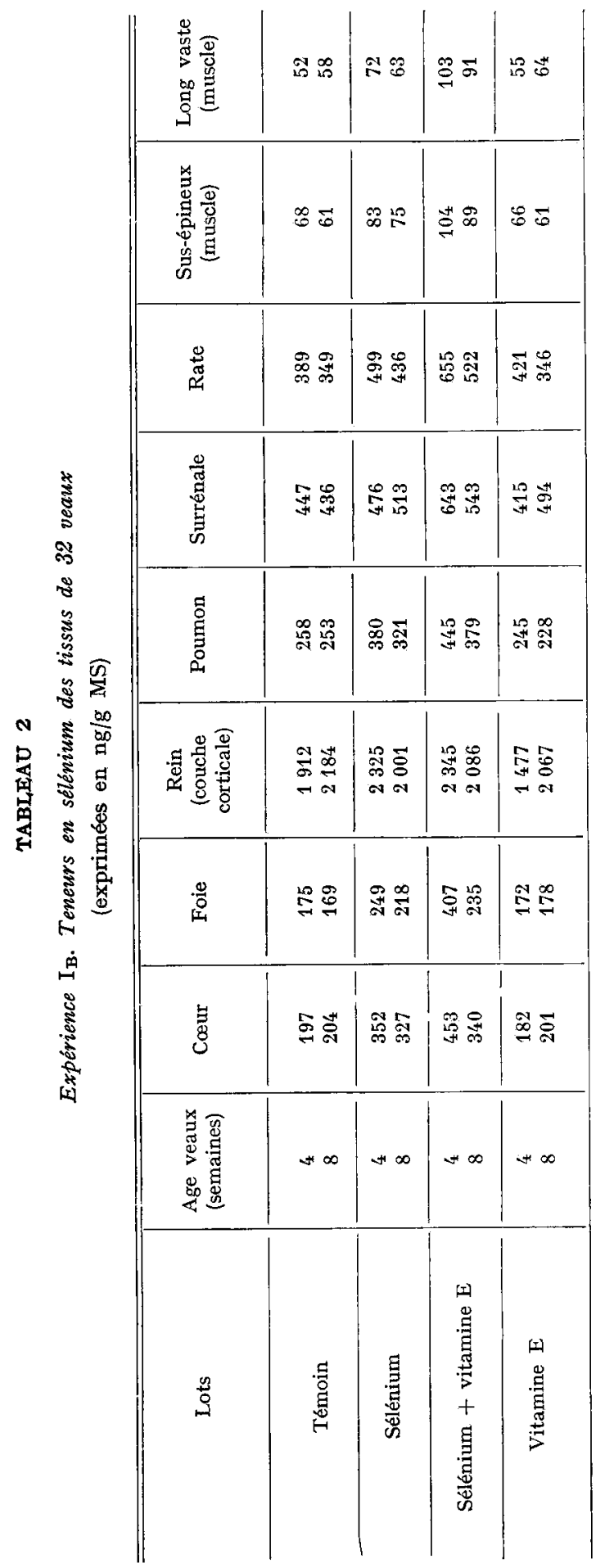

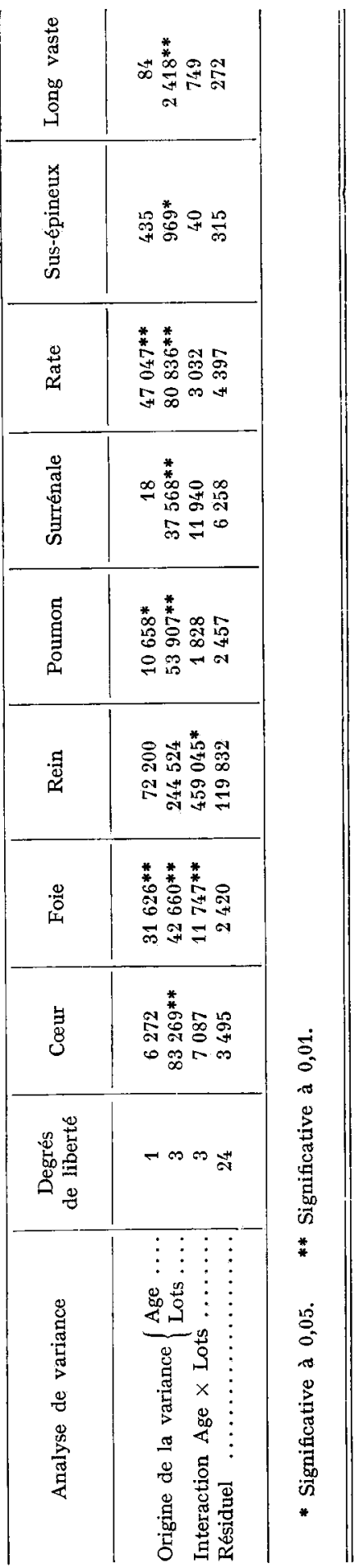




\section{Résultats et discussion}

Les résultats sont consignés dans le tableau 3. La différence en teneur de sélénium entre les divers lots est significative pour les tissus ci-après :

$$
\begin{aligned}
& \text { foie, poumon }(P=0,05) \\
& \text { rate, cœur } \quad(P=0,01)
\end{aligned}
$$

Ces teneurs sont supérieures chez les agneaux nés de brebis implantées au sélénium. Chez ces mêmes agneaux, si les concentrations de ce micro-élément dans les différents tissus sont légèrement plus élevées à 60 jours qu'à I20 jours, en fait les différences n'étaient pas significatives $(P<0,05)$. En conséquence, l'effet de 1'âge a été ignoré lors des calculs.

\section{TABLEAU 3}

Expérience $2_{\mathrm{A}}$. Teneurs en sélénium des tissus de 18 agneaux nés des brebis ci-dessous (ng/g MS)

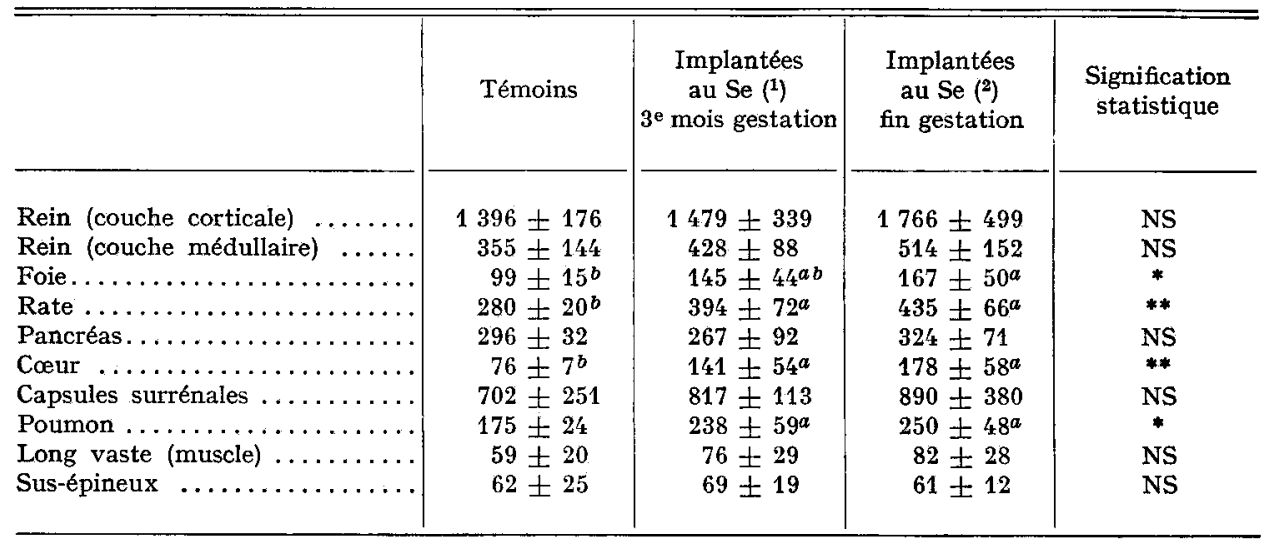

$* \mathrm{P}<0,05$.

** $\mathrm{P}<0,01$.

(1) Dans chaque groupe, 2 agneaux âgés respectivement de 60,90 et 120 jours ont été abattus. Les différences dans la concentration du sélénium n'ayant pas été influencées par l'áge, les teneurs en sélénium tissulaire ont été groupées pour chaque lot.

(2) $20 \mathrm{mg}$ sélénium sous forme de sélénite de sodium.

Chez tous les agneaux mentionnés dans la présente étude, les teneurs en sélénium tissulaire sont bien inférieures à celles observées dans une autre étude où les agneaux étaient nés de brebis nourries au foin de la région d'Ottawa et supplémentées au grain de l'ouest canadien qui contenait $400 \mathrm{ng}$ de Se par g de MS (HIDIRogr,ou et al., I968).

Afin de s'assurer de la salubrité de la viande de nos agneaux nés de brebis implantées au sélénium, nous avons pensé qu'une comparaison avec les résultats rapportés sur les teneurs en sélénium tissulaire des agneaux par d'autres auteurs était nécessaire. Ainsi, sur des agneaux destinés à l'engraissement et nourris aux comprimés contenant I45 ng/g de MS, LINDBERG et JACOBSON (I970) ont rapporté les chiffres 
ci-après : Foie 647, Rein $6880 \mathrm{ng} / \mathrm{g}$ de MS. Cousins et CAIRNEY (I96I), sur des agneaux allaités, sains, rapportaient : Foie 280, Rein $2220 \mathrm{ng} / \mathrm{g}$ de MS et sur des agneaux nourris aux comprimés : Foie 800 et Rein $3700 \mathrm{ng} / \mathrm{g}$ de MS. D'autre part, PAULson et BRODERICK (I968) ayant mis à la disposition des brebis et de leurs agneaux un composé minéral contenant 26 p.p.m. de Se ont rapporté que les reins et le foie contenaient 4330 et $930 \mathrm{ng} / \mathrm{g}$ de $\mathrm{MS}$.

Ces derniers auteurs, en comparant ces teneurs avec celles trouvées dans d'autres régions des États-Unis, concluent que la viande de ces agneaux est comestible.

Les valeurs précitées sur les teneurs en sélénium proviennent d'animaux sains et il est à remarquer qu'elles sont supérieures aux nôtres.

Il en ressort donc que tout en bénéficiant de l'implantation de sélénium effectuée sur leurs mères, la viande de nos agneaux reste comestible, n'accumulant pas un excès de sélénium.

\section{EXPÉRIENCE SUR BREBIS $2_{\text {B }}$}

\section{Matériel et méthodes}

Vingt-deux brebis de race Shropshire ont été utilisées.

Seize animaux provenaient d'un lot de brebis nourries depuis 9 mois avec un foin dont la teneur en sélénium était de $25 \mathrm{ng} / \mathrm{g}$ ed MS, alors que les 6 autres brebis (lot 3 ) étaient alimentées avec un foin de $70 \mathrm{ng} / \mathrm{g}$ de $\mathrm{MS}$, lequel était supplémenté avec du grain à $270 \mathrm{ng} / \mathrm{g}$ de $\mathrm{MS}$.

Les 16 premiers animaux ont été répartis en 2 lots de huit chacun.

Lot I Témoins.

Lot 2 Brebis implantées avec une préparation (44 $\mathrm{mg}$ ) contenant du sélénium ( $22 \mathrm{mg}$ ) sous forme de sélénite de sodium et d'une colle aux silicones. Le lieu d'implantation était la région sus-sternale, laquelle avait été au préalable tatouée en vue de reconnaître le lieu d'implantation. tivement.

Deux animaux des groupes I et $2(56-65 \mathrm{~kg})$ ont été abattus à $2,3,4$ et 8 semaines respec.

Les 6 autres brebis du lot 3 ont également été tuées et les différents tissus de tous les animaux ont été analysés en vue de déterminer leur teneur en sélénium.

\section{Résultats et discussion}

Les concentrations en sélénium tissulaire sont inférieures chez les sujets nourris au foin ayant une faible teneur en sélénium (lot I), que chez les animaux des 2 autres lots ( 2 et 3 ) implantés au sélénium ou nourris au grain. On peut cependant noter que dans ces deux derniers lots, les résultats ne sont pas différents (tabl. 4).

D'une façon générale, chez les sujets implantés, les teneurs tissulaires en sélénium sont du même ordre de grandeur que celles rapportées par BuRToN et al. (I962) sur des brebis nourries avec un foin non-carencé en sélénium. Par contre, dans le lot $\mathrm{I}$, les teneurs en sélénium des tissus sont similaires à celles des brebis (BURTON et al., I962) ayant donné naissance à des agneaux atteints de DM.

Chez les sujets implantés, il convient de souligner que pendant l'expérience de ces 2 mois, la comparaison des taux extrêmes de sélénium ne fait pas ressortir une diminution notable de ce micro-élément. Ce maintien était probablement lié à l'effet continu et prolongé de l'utilisation du sélénium implanté sur la brebis nourrie au foin pauvre en sélénium. 
TABLEAU 4

Expérience 28. Teneurs en sélénium des tissus

(ng/g MS) des brebis ci-dessous

\begin{tabular}{|c|c|c|c|c|}
\hline Tissus & $\begin{array}{c}\text { Lot } 1 \\
\text { Au foin } \\
\text { produisant } \\
\text { la dystrophie } \\
\text { (8) DM }\end{array}$ & $\begin{array}{c}\text { Lot } 2 \\
\text { Au foin } \\
\text { produisant DM } \\
\text { mais } \\
\text { Se-implantées } \\
\text { (8) : } 22 \mathrm{mg}\end{array}$ & $\begin{array}{c}\text { Lot } 3 \\
\text { Au foin et grain } \\
(6)\end{array}$ & $\begin{array}{l}\text { Signification } \\
\text { statistique }\end{array}$ \\
\hline Rein (couche corticale) & $2959 \pm 493^{b}$ & $5001+547^{a}$ & $3995+781^{a}$ & * \\
\hline Rein (couche médullaire) & $619 \pm 260^{b}$ & $1537 \pm 623^{a}$ & $1230 \pm 333^{a}$ & * \\
\hline Foie $\ldots \ldots \ldots \ldots \ldots \ldots$ & $369 \pm 130^{b}$ & $1031 \pm 310^{a}$ & $658 \pm 106^{a}$ & * \\
\hline Rate $\ldots \ldots \ldots \ldots \ldots$. & $548 \pm 124^{b}$ & $1045 \pm 160^{a}$ & $1082 \pm 143^{a}$ & ** \\
\hline Cour $\ldots \ldots \ldots \ldots \ldots \ldots$ & $282 \pm 111^{b}$ & $799 \pm 96^{a}$ & $693 \pm 165^{a}$ & ** \\
\hline Poumon $\ldots \ldots \ldots \ldots \ldots \ldots$ & $361 \pm 71^{b}$ & $930 \pm 186^{a}$ & $829 \pm 79^{a}$ & ** \\
\hline Long vaste (muscle) .......... & $96 \pm 31^{b}$ & $189 \pm 67^{b}$ & $235 \pm 49^{a}$ & $* *$ \\
\hline Sus-épineux (muscle) $\ldots \ldots \ldots$ & $55 \pm 21^{b}$ & $132 \pm 21^{a}$ & 二 & $* *$ \\
\hline
\end{tabular}

* $\mathrm{P}<0,65$

** $P<0,01$. ( ) nombre brebis (poids 55 à $65 \mathrm{~kg}$ ).

En ligne horizontale : les valeurs affectées de la même lettre ne sont pas significativement différentes entre elles.

Il est intéressant de constater l'importante rétention de sélénium au lieu d'implantation. En effet, au lieu d'implantation, sur un échantillon de $30 \mathrm{~g}$ prélevé sur toutes les brebis implantées, on a observé des valeurs de 2 à $4 \mathrm{mg}$ de sélénium, et sur les 2 brebis tuées 2 mois après, c'est-à-dire à la fin de l'expérience, on a encore trouvé $\mathrm{I}, 6$ à $\mathrm{x}, 8 \mathrm{mg}$.

En conclusion, la brebis soumise à ce mode de traitement sélénique retient et utilise cet élément d'une manière normale et continue, sans accumulation excessive dans ses différents organes. En revanche, au lieu d'implantation, le sélénium a été retenu longtemps, ce qui rend la viande de cette brebis insalubre.

Par ailleurs, dans cette expérience, le sélénium ayant été implanté dans la région sus-sternale, 1'inconvénient majeur de cette technique pourrait être corrigé en cherchant d'autres lieux d'implantation susceptibles d'être enlevés avant l'abattage (par ex. queue, oreille).

Reçu pour publication en juin 1971.

\section{SUMMARY}

\section{SELENIUM CONTENT IN TISSUES OF RUMINANTS}

The aim of the study was to find the content of selenium in tissues of ruminants after they had been given this trace element.

In a pilot study with $\mathrm{I}_{4}$ bullocks, 6 were given a single dose of $30 \mathrm{mg}$ selenium by muscle, 6 got 2 doses and 2 got none. Meat from the cattle killed 6 weeks after the single dose was hygenically satisfactory. For those given 2 doses a lapse of 15 weeks was necessary. 
In another experiment 32 calves were in 4 groups of 8 . For 2 groups $4 \mathrm{mg}$ selenium was given by mouth alone or with vitamin $\mathrm{E}$. Of the other groups one got vitamin $\mathrm{E}$ and the other was not treated. With treatment with selenium the calves could be freed for human consumption after 4 weeks.

Selenium was estimated in tissues of I 8 lambs from ewes not treated or given selenium in a silicone paste by implant. The results showed that this new method of giving selenium prevented muscular dystrophy while not affecting the wholesomeness of the meat of the lambs.

Selenium was estimated in tissues of 16 ewes given selenium by implant and fed on hay with low selenium content and in 6 normal ewes on a diet of hay and concentrate with normal selenium content. There was no difference in selenium content of the different tissues between treated and normal ewes. There was some accumulation at the site of implant in the ewes given it, even after 2 months.

\section{REMERCIEMEN'TS}

Nous remercions vivement le personnel de la ferme expérimentale de Kapuskasing ainsi que celui du Service de Recherches de Chimie analytique pour l'aide technique qu'ils nous ont apportée.

\section{RÉFÉRENCES BIBLIOGRAPHIQUES}

Burton V., Keeler R. F., Swingle K. F, Young S., r962. Nutritional muscular distrophy in lambs Selenium analysis of maternal and juvenile tissues. 'Am. J. Vet. Res., 23, 962-965.

Cousins F. B., Cairney I. M., 196r. Some aspects of selenium metabolism in sheep. Aust. J.Agric. Res., 12, 927-943.

DoIG P. A., I970. Muscular distrophy in yearling cattle. Can. Vet. Jour., 11, 24-25.

Decale J. P., I969. Myopathie dégénérative chez les bovins de race Limousine. Thèse Doct. Vét. Alfort.

Dotta U., Balbo T., Guarda F., x 964 . Saggi comparatavi sulle proprieta terapeutiche del " selenio" e della "vitamina "nella miodistrofia enzootica dei vitelli, Prog. Veter., 19, 38-52.

Flachat Ch., Van Havarbeke G., chantegrelet G., Dufour C., I967. Dégénérescence musculaire es jeunes bovins précoces. Revue Méd. Vét., 11, 863-882.

Hidiroglou M., Jenkins K., Carson R. B., Brossard, C. A., I967. Selenium and coenzyme $Q_{10}$ levels $n$ the tissue of dystrophic and healthy calves. Can. J. Physiol. Pharmac., 45, 658-659.

Hidiroglov M., Jenkins K. J., Carson R. B., Mac Kay R. R., I968. Some aspects of selenium metabolism in normal an dystrophic sheep. Can. J. Anim. Sci., 48, 335-346.

Hidiroglou M., Jenkins K., Carson R. B., 1969. Considerations sur les métabolismes du sélénium et de la vitamine $\mathrm{E}$ chez les jeunes ruminants. Ann. Biol. anim. Bioch. Biochim., 9, I6I-I7o.

Hidroglou M., Hoffman I., Jenkins K., 197I. Control of Nutritional muscular dystrophy in lambs by selenium implantation. Animal Prod. (sous presse).

Hoffman I., Westerby R. J., Hidiroglou M., rg68. Precise fluorometric microdetermination of selenium in agricultural materials. J.A.O.A.C., 51, 1039-1042.

LAmand M., 1965. Syndrome myopathie-dyspnée. Influence de la prophylaxie par le sélénium sur la composition biochimique du plasma chez le veau. Ann. Biol, anim. Bioch. Biophys., 5, 309-315.

LiNdBERg P., I96. Selenium determination in plant and animal material, and in water. Acta Vet. Scand. suppl. 23.

LindBerg P., Jacosson S. O., r97o. Relationship between selenium content of forage, blood and organs of sheep and mortality rate. Acta Vet. Scand., 11, 49-58.

MaAg D. D., GlenN M. W., r967. Toxicity of selenium : farm animals p. I27-I40 in Symposium Selenium in Biomedicine. Ed. Muth, O. H. The Avi Publishing Co., Westport, Connecticut.

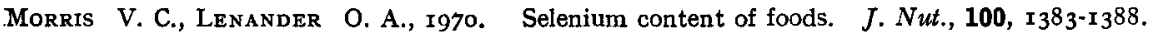

Paulson G. D., Broderick G. A., r968. Effect of feeding sheep selenium fortified trace mineralized salt : effect of tocopherol. Anim. Sci., 27, 195-202.

Sutherland G. L., 1969. Principles of establishing withdrawal periods for feeds containing drugs, 244-254. Coll. The use of drugs in animal feeds. National Academy Sciences Publ. r 79 . Washington, D. C. 